DESY 07-069

May 2007

\title{
Bose-Einstein Correlations of Charged and Neutral Kaons in Deep Inelastic Scattering at HERA
}

ZEUS Collaboration

\begin{abstract}
Bose-Einstein correlations of charged and neutral kaons have been measured in $e^{ \pm} p$ deep inelastic scattering with an integrated luminosity of $121 \mathrm{pb}^{-1}$ using the ZEUS detector at HERA. The two-particle correlation function was studied as a function of the four-momentum difference of the kaon pairs, $Q_{12}=$ $\sqrt{-\left(\mathrm{p}_{1}-\mathrm{p}_{2}\right)^{2}}$, assuming a Gaussian shape for the particle source. The values of the radius of the production volume, $r$, and of the correlation strength, $\lambda$, were obtained for both neutral and charged kaons. The radii for charged and neutral kaons are similar and are consistent with those obtained at LEP.
\end{abstract}




\section{The ZEUS Collaboration}

S. Chekanov'ㄹ, M. Derrick, S. Magill, B. Musgrave, D. Nicholass'², J. Repond, R. Yoshida Argonne National Laboratory, Argonne, Illinois 60439-4815, USA ${ }^{n}$

M.C.K. Mattingly

Andrews University, Berrien Springs, Michigan 49104-0380, USA

M. Jechow, N. Pavel ${ }^{\dagger}$, A.G. Yagües Molina

Institut für Physik der Humboldt-Universität zu Berlin, Berlin, Germany

S. Antonelli, P. Antonioli, G. Bari, M. Basile, L. Bellagamba, M. Bindi, D. Boscherini,

A. Bruni, G. Bruni, L. Cifarelli, F. Cindolo, A. Contin, M. Corradi, S. De Pasquale,

G. Iacobucci, A. Margotti, R. Nania, A. Polini, G. Sartorelli, A. Zichichi

University and INFN Bologna, Bologna, Italy ${ }^{e}$

D. Bartsch, I. Brock, S. Goers ${ }^{3}$, H. Hartmann, E. Hilger, H.-P. Jakob, M. Jüngst, O.M. Kind ${ }^{4}$, A.E. Nuncio-Quiroz, E. Paul ${ }^{5}$, R. Renner ${ }^{6}$, U. Samson, V. Schönberg, R. Shehzadi, M. Wlasenko Physikalisches Institut der Universität Bonn, Bonn, Germany ${ }^{b}$

N.H. Brook, G.P. Heath, J.D. Morris, T. Namsoo

H.H. Wills Physics Laboratory, University of Bristol, Bristol, United Kingdom ${ }^{m}$

M. Capua, S. Fazio, A. Mastroberardino, M. Schioppa, G. Susinno, E. Tassi

Calabria University, Physics Department and INFN, Cosenza, Italy ${ }^{e}$

J.Y. $\operatorname{Kim}^{7}$, K.J. Ma ${ }^{8}$

Chonnam National University, Kwangju, South Korea ${ }^{g}$

Z.A. Ibrahim, B. Kamaluddin, W.A.T. Wan Abdullah

Jabatan Fizik, Universiti Malaya, 50603 Kuala Lumpur, Malaysia ${ }^{r}$

Y. Ning, Z. Ren, F. Sciulli

Nevis Laboratories, Columbia University, Irvington on Hudson, New York 10027o

J. Chwastowski, A. Eskreys, J. Figiel, A. Galas, M. Gil, K. Olkiewicz, P. Stopa, L. Zawiejski

The Henryk Niewodniczanski Institute of Nuclear Physics, Polish Academy of Sciences, Cracow, Poland ${ }^{i}$

L. Adamczyk, T. Bołd, I. Grabowska-Bołd, D. Kisielewska, J. Łukasik, M. Przybycień,

L. Suszycki

Faculty of Physics and Applied Computer Science, AGH-University of Science and Technology, Cracow, Poland ${ }^{p}$

A. Kotański ${ }^{9}$, W. Słomiński ${ }^{10}$

Department of Physics, Jagellonian University, Cracow, Poland 
V. Adler ${ }^{11}$, U. Behrens, I. Bloch, C. Blohm, A. Bonato, K. Borras, R. Ciesielski, N. Coppola, A. Dossanov, V. Drugakov, J. Fourletova, A. Geiser, D. Gladkov, P. Göttlicher ${ }^{12}$, J. Grebenyuk, I. Gregor, T. Haas, W. Hain, C. Horn ${ }^{13}$, A. Hüttmann, B. Kahle, I.I. Katkov, U. Klein ${ }^{14}$, U. Kötz, H. Kowalski, E. Lobodzinska, B. Löhr, R. Mankel, I.-A. MelzerPellmann, S. Miglioranzi, A. Montanari, D. Notz, L. Rinaldi, P. Roloff, I. Rubinsky, R. Santamarta, U. Schneekloth, A. Spiridonov ${ }^{15}$, H. Stadie, D. Szuba ${ }^{16}$, J. Szuba ${ }^{17}$, T. Theedt, G. Wolf, K. Wrona, C. Youngman, W. Zeuner Deutsches Elektronen-Synchrotron DESY, Hamburg, Germany

W. Lohmann, S. Schlenstedt

Deutsches Elektronen-Synchrotron DESY, Zeuthen, Germany

G. Barbagli, E. Gallo, P. G. Pelfer

University and INFN, Florence, Italy ${ }^{e}$

A. Bamberger, D. Dobur, F. Karstens, N.N. Vlasov ${ }^{18}$

Fakultät für Physik der Universität Freiburg i.Br., Freiburg i.Br., Germany ${ }^{b}$

P.J. Bussey, A.T. Doyle, W. Dunne, J. Ferrando, M. Forrest, D.H. Saxon, I.O. Skillicorn

Department of Physics and Astronomy, University of Glasgow, Glasgow, United Kingdom $^{m}$

I. Gialas ${ }^{19}$, K. Papageorgiu

Department of Engineering in Management and Finance, Univ. of Aegean, Greece

T. Gosau, U. Holm, R. Klanner, E. Lohrmann, H. Salehi, P. Schleper, T. Schörner-Sadenius, J. Sztuk, K. Wichmann, K. Wick

Hamburg University, Institute of Exp. Physics, Hamburg, Germany ${ }^{b}$

C. Foudas, C. Fry, K.R. Long, A.D. Tapper

Imperial College London, High Energy Nuclear Physics Group, London, United Kingdom $^{m}$

M. Kataoka ${ }^{20}$, T. Matsumoto, K. Nagano, K. Tokushuku ${ }^{21}$, S. Yamada, Y. Yamazaki Institute of Particle and Nuclear Studies, KEK, Tsukuba, Japan ${ }^{f}$

A.N. Barakbaev, E.G. Boos, N.S. Pokrovskiy, B.O. Zhautykov

Institute of Physics and Technology of Ministry of Education and Science of Kazakhstan, Almaty, Kazakhstan

V. Aushev ${ }^{1}$

Institute for Nuclear Research, National Academy of Sciences, Kiev and Kiev National University, Kiev, Ukraine

D. Son

Kyungpook National University, Center for High Energy Physics, Daegu, South Korea ${ }^{g}$ 
J. de Favereau, K. Piotrzkowski

Institut de Physique Nucléaire, Université Catholique de Louvain, Louvain-la-Neuve, Belgium $^{q}$

F. Barreiro, C. Glasman²2, M. Jimenez, L. Labarga, J. del Peso, E. Ron, M. Soares, J. Terrón, M. Zambrana

Departamento de Física Teórica, Universidad Autónoma de Madrid, Madrid, Spain ${ }^{l}$

F. Corriveau, C. Liu, R. Walsh, C. Zhou

Department of Physics, McGill University, Montréal, Québec, Canada H3A 2T8 ${ }^{a}$

T. Tsurugai

Meiji Gakuin University, Faculty of General Education, Yokohama, Japan ${ }^{f}$

A. Antonov, B.A. Dolgoshein, V. Sosnovtsev, A. Stifutkin, S. Suchkov

Moscow Engineering Physics Institute, Moscow, Russia ${ }^{j}$

R.K. Dementiev, P.F. Ermolov, L.K. Gladilin, L.A. Khein, I.A. Korzhavina, V.A. Kuzmin,

B.B. Levchenko ${ }^{23}$, O.Yu. Lukina, A.S. Proskuryakov, L.M. Shcheglova, D.S. Zotkin, S.A. Zotkin

Moscow State University, Institute of Nuclear Physics, Moscow, Russia ${ }^{k}$

I. Abt, C. Büttner, A. Caldwell, D. Kollar, W.B. Schmidke, J. Sutiak

Max-Planck-Institut für Physik, München, Germany

G. Grigorescu, A. Keramidas, E. Koffeman, P. Kooijman, A. Pellegrino, H. Tiecke, M. Vázquez ${ }^{20}$, L. Wiggers

NIKHEF and University of Amsterdam, Amsterdam, Netherlands ${ }^{h}$

N. Brümmer, B. Bylsma, L.S. Durkin, A. Lee, T.Y. Ling

Physics Department, Ohio State University, Columbus, Ohio 43210 ${ }^{n}$

P.D. Allfrey, M.A. Bell, A.M. Cooper-Sarkar, A. Cottrell, R.C.E. Devenish, B. Foster, K. Korcsak-Gorzo, S. Patel, V. Roberfroid ${ }^{24}$, A. Robertson, P.B. Straub, C. UribeEstrada, R. Walczak

Department of Physics, University of Oxford, Oxford United Kingdom ${ }^{m}$

P. Bellan, A. Bertolin, R. Brugnera, R. Carlin, F. Dal Corso, S. Dusini, A. Garfagnini,

S. Limentani, A. Longhin, L. Stanco, M. Turcato

Dipartimento di Fisica dell' Università and INFN, Padova, Italy ${ }^{e}$

B.Y. Oh, A. Raval, J. Ukleja ${ }^{25}$, J.J. Whitmore ${ }^{26}$

Department of Physics, Pennsylvania State University, University Park, Pennsylvania $16802^{\circ}$

Y. Iga

Polytechnic University, Sagamihara, Japan ${ }^{f}$ 
G. D'Agostini, G. Marini, A. Nigro

Dipartimento di Fisica, Università 'La Sapienza' and INFN, Rome, Italy ${ }^{e}$

J.E. Cole, J.C. Hart

Rutherford Appleton Laboratory, Chilton, Didcot, Oxon, United Kingdom ${ }^{m}$

H. Abramowicz ${ }^{27}$, A. Gabareen, R. Ingbir, S. Kananov, A. Levy

Raymond and Beverly Sackler Faculty of Exact Sciences, School of Physics, Tel-Aviv University, Tel-Aviv, Israel ${ }^{d}$

M. Kuze, J. Maeda

Department of Physics, Tokyo Institute of Technology, Tokyo, Japan ${ }^{f}$

R. Hori, S. Kagawa ${ }^{28}$, N. Okazaki, S. Shimizu, T. Tawara

Department of Physics, University of Tokyo, Tokyo, Japan ${ }^{f}$

R. Hamatsu, H. Kaji ${ }^{29}$, S. Kitamura ${ }^{30}$, O. Ota, Y.D. Ri

Tokyo Metropolitan University, Department of Physics, Tokyo, Japan ${ }^{f}$

M.I. Ferrero, V. Monaco, R. Sacchi, A. Solano

Università di Torino and INFN, Torino, Italy ${ }^{e}$

M. Arneodo, M. Ruspa

Università del Piemonte Orientale, Novara, and INFN, Torino, Italy ${ }^{e}$

S. Fourletov, J.F. Martin

Department of Physics, University of Toronto, Toronto, Ontario, Canada M5S 1A7 a

S.K. Boutle ${ }^{19}$, J.M. Butterworth, C. Gwenlan ${ }^{31}$, T.W. Jones, J.H. Loizides, M.R. Sutton ${ }^{31}$, M. Wing

Physics and Astronomy Department, University College London, London, United Kingdom $^{m}$

B. Brzozowska, J. Ciborowski ${ }^{32}$, G. Grzelak, P. Kulinski, P. Łużniak ${ }^{33}$, J. Malka ${ }^{33}$, R.J. Nowak, J.M. Pawlak, T. Tymieniecka, A. Ukleja, A.F. Żarnecki

Warsaw University, Institute of Experimental Physics, Warsaw, Poland

M. Adamus, P. Plucinski ${ }^{34}$

Institute for Nuclear Studies, Warsaw, Poland

Y. Eisenberg, I. Giller, D. Hochman, U. Karshon, M. Rosin

Department of Particle Physics, Weizmann Institute, Rehovot, Israel ${ }^{c}$

E. Brownson, T. Danielson, A. Everett, D. Kçira, D.D. Reeder ${ }^{5}$, P. Ryan, A.A. Savin, W.H. Smith, H. Wolfe

Department of Physics, University of Wisconsin, Madison, Wisconsin 53706, USA ${ }^{n}$

S. Bhadra, C.D. Catterall, Y. Cui, G. Hartner, S. Menary, U. Noor, J. Standage, J. Whyte Department of Physics, York University, Ontario, Canada M3J 1P3 ${ }^{a}$ 
${ }^{1}$ supported by DESY, Germany

2 also affiliated with University College London, UK

${ }^{3}$ now with TÜV Nord, Germany

${ }^{4}$ now at Humboldt University, Berlin, Germany

${ }^{5}$ retired

${ }^{6}$ self-employed

7 supported by Chonnam National University in 2005

${ }^{8}$ supported by a scholarship of the World Laboratory Björn Wiik Research Project

9 supported by the research grant no. 1 P03B 04529 (2005-2008)

10 This work was supported in part by the Marie Curie Actions Transfer of Knowledge project COCOS (contract MTKD-CT-2004-517186)

11 now at Univ. Libre de Bruxelles, Belgium

12 now at DESY group FEB, Hamburg, Germany

13 now at Stanford Linear Accelerator Center, Stanford, USA

14 now at University of Liverpool, UK

15 also at Institut of Theoretical and Experimental Physics, Moscow, Russia

16 also at INP, Cracow, Poland

17 on leave of absence from FPACS, AGH-UST, Cracow, Poland

18 partly supported by Moscow State University, Russia

19 also affiliated with DESY

20 now at CERN, Geneva, Switzerland

21 also at University of Tokyo, Japan

22 Ramón y Cajal Fellow

23 partly supported by Russian Foundation for Basic Research grant no. 05-02-39028NSFC-a

${ }^{24}$ EU Marie Curie Fellow

25 partially supported by Warsaw University, Poland

26 This material was based on work supported by the National Science Foundation, while working at the Foundation.

27 also at Max Planck Institute, Munich, Germany, Alexander von Humboldt Research Award

28 now at KEK, Tsukuba, Japan

${ }^{29}$ now at Nagoya University, Japan

30 Department of Radiological Science

31 PPARC Advanced fellow

32 also at Łódź University, Poland

33 Łódź University, Poland

${ }^{34}$ supported by the Polish Ministry for Education and Science grant no. 1 P03B 14129

$\dagger$ deceased 
a supported by the Natural Sciences and Engineering Research Council of Canada (NSERC)

$b$ supported by the German Federal Ministry for Education and Research (BMBF), under contract numbers HZ1GUA 2, HZ1GUB 0, HZ1PDA 5, HZ1VFA 5

c supported in part by the MINERVA Gesellschaft für Forschung GmbH, the Israel Science Foundation (grant no. 293/02-11.2) and the U.S.-Israel Binational Science Foundation

$d \quad$ supported by the German-Israeli Foundation and the Israel Science Foundation

$e$ supported by the Italian National Institute for Nuclear Physics (INFN)

$f$ supported by the Japanese Ministry of Education, Culture, Sports, Science and Technology (MEXT) and its grants for Scientific Research

$g$ supported by the Korean Ministry of Education and Korea Science and Engineering Foundation

$h$ supported by the Netherlands Foundation for Research on Matter (FOM)

$i$ supported by the Polish State Committee for Scientific Research, grant no. 620/E-77/SPB/DESY/P-03/DZ 117/2003-2005 and grant no. 1P03B07427/2004-2006

j partially supported by the German Federal Ministry for Education and Research $(\mathrm{BMBF})$

$k$ supported by RF Presidential grant N 8122.2006.2 for the leading scientific schools and by the Russian Ministry of Education and Science through its grant Research on High Energy Physics

$l$ supported by the Spanish Ministry of Education and Science through funds provided by CICYT

$m$ supported by the Particle Physics and Astronomy Research Council, UK

$n$ supported by the US Department of Energy

$o$ supported by the US National Science Foundation. Any opinion, findings and conclusions or recommendations expressed in this material are those of the authors and do not necessarily reflect the views of the National Science Foundation.

$p$ supported by the Polish Ministry of Science and Higher Education as a scientific project (2006-2008)

$q$ supported by FNRS and its associated funds (IISN and FRIA) and by an Inter-University Attraction Poles Programme subsidised by the Belgian Federal Science Policy Office

$r$ supported by the Malaysian Ministry of Science, Technology and Innovation/Akademi Sains Malaysia grant SAGA 66-02-03-0048 


\section{Introduction}

The use of Bose-Einstein correlations (BEC) in particle physics as a method of determininig the size and the shape of the source from which particles originate was first considered by Goldhaber et al. [1,2] in 1959 for $p \bar{p}$ annihilations. Bose-Einstein correlations originate from the symmetrization of the two-particle wave function of identical bosons and lead to an enhancement of boson pairs emitted with small relative momenta. The effect is sensitive to the size of the emitting source. The studies of BEC for pairs of identical particles have been carried out in a large variety of particle interactions. In particular, H1 and ZEUS have reported results on inclusive charged particle pairs in $e^{ \pm} p$ collisions at HERA $[3,4]$. Recent reviews [5-9] summarise the underlying theoretical concepts and experimental results. The measurements of the radius of the emission source have been mostly performed for neutral and charged pions. For other bosons, e.g. kaons, the information is scanty.

This paper reports first results on BEC for pairs of charged $\left(K^{ \pm} K^{ \pm}\right)$and neutral $\left(K_{S}^{0} K_{S}^{0}\right)$ kaons in deep inelastic scattering (DIS) at HERA. The measurements are compared with results from $e^{+} e^{-}$interactions whose fragmentation properties are expected to be similar to the current region of DIS [10]. However, proton fragmentation may lead to a significant difference in the properties of the hadronic final state.

The correlation function for two identical kaons is defined as

$$
R\left(Q_{12}\right)=\frac{P\left(Q_{12}\right)}{P_{0}\left(Q_{12}\right)}
$$

where $Q_{12}$ is the four-momenta difference of the kaons with four momenta $\mathrm{p}_{1}$ and $\mathrm{p}_{2}$ given as

$$
Q_{12}=\sqrt{-\left(\mathrm{p}_{1}-\mathrm{p}_{2}\right)^{2}}=\sqrt{M_{K K}^{2}-4 m_{K}^{2}},
$$

where $M_{K K}$ is the invariant mass of the pair of kaons and $m_{K}$ is the kaon rest mass. The function $P\left(Q_{12}\right)$ in Eq. (1) is the two-particle density: $P\left(Q_{12}\right)=(1 / N)\left(d n_{K K} / d Q_{12}\right)$, where $n_{K K}$ is the number of kaon pairs and $N$ is the number of events. The denominator of Eq. (1), $P_{0}\left(Q_{12}\right)$, is the two-particle density in the absence of BEC.

For a static source with a Gaussian density distribution, the correlation function can be parametrised as follows [2]:

$$
R\left(Q_{12}\right)=1+\lambda \exp \left(-r^{2} Q_{12}^{2}\right)
$$


The $\lambda$ parameter gives information about the strength of the BEC. For a completely coherent source, $\lambda$ is zero, while for a completely incoherent source, $\lambda$ is one [11]. Contributions from decays of short-lived resonances can further modify this parameter. The parameter $r$ is related to the size of the source and is called the radius in the following sections.

\section{Experimental set-up}

The analysis was performed with data taken by the ZEUS detector between 1996 and 2000 at HERA. The data from $e^{ \pm} p$ collisions collected in this period with electron ${ }^{1}$ energy $E_{e}=$ $27.5 \mathrm{GeV}$ and proton energy $E_{p}=820 \mathrm{GeV}(1996-1997)$ or $E_{p}=920 \mathrm{GeV}(1998-2000)$ correspond to an integrated luminosity of $121 \mathrm{pb}^{-1}$.

A detailed description of the ZEUS detector can be found elsewhere [12]. A brief outline of the components that are most relevant for this analysis is given below.

Charged particles are tracked in the central tracking detector (CTD) [13], which operates in a magnetic field of $1.43 \mathrm{~T}$ provided by a thin superconducting coil. The CTD consists of 72 cylindrical drift chamber layers, organized in 9 superlayers covering the polarangle $^{2}$ region $15^{\circ}<\theta<164^{\circ}$. The transverse-momentum resolution for full-length tracks is $\sigma\left(p_{T}\right) / p_{T}=0.0058 p_{T} \oplus 0.0065 \oplus 0.0014 / p_{T}$, with $p_{T}$ in $\mathrm{GeV}$. To estimate the energy loss, $d E / d x$, of tracks, the truncated mean of the sense-wire pulse-heights was recorded for each track, discarding the $10 \%$ lowest and up to the $30 \%$ highest pulses [14-16]. The measured $d E / d x$ values were normalised to the $d E / d x$ peak position for tracks with momenta $0.3<p<0.4 \mathrm{GeV}$, the region of minimum ionisation for pions. Henceforth, $d E / d x$ is quoted in units of minimum ionising particles (mips). The resolution of the $d E / d x$ measurement for full-length tracks is about $9 \%$. The tracking system was used to establish the primary and secondary vertices.

The high-resolution uranium-scintillator calorimeter (CAL) [17] consists of three parts: the forward, the barrel and the rear (RCAL) calorimeters. Each part is subdivided transversely into towers and longitudinally into one electromagnetic section and either one or two hadronic sections. The smallest subdivision of the calorimeter is called a cell. The CAL energy resolutions, as measured under test-beam conditions, are $\sigma(E) / E=0.18 / \sqrt{E}$ for electrons and $\sigma(E) / E=0.35 / \sqrt{E}$ for hadrons, with $E$ in $\mathrm{GeV}$.

\footnotetext{
${ }^{1}$ Here and in the following, the term „electron" denotes generically both the electron and the positron.

2 The ZEUS coordinate system is a right-handed Cartesian system, with the $Z$ axis pointing in the proton beam direction, referred to as the "forward direction", and the $X$ axis pointing left towards the center of HERA. The coordinate origin is at the nominal interaction point.
} 
The energy of the scattered electron was corrected for energy loss in the material between the interaction point and the calorimeter using the small-angle rear tracking detector $[18,19]$ and the presampler $[18,20]$.

\section{Event and track selection}

The inclusive neutral current DIS process $e(k)+p(P) \rightarrow e\left(k^{\prime}\right)+X$ can be described in terms of the following kinematic variables: $Q^{2}$, the virtuality of the exchanged photon, $x$, the Bjorken scaling variable and $y$, the fraction of the lepton energy transferred to the proton in the proton rest frame. They are defined as follows: $Q^{2}=-q^{2}=-\left(k-k^{\prime}\right)^{2}$; $x=Q^{2} /(2 P \cdot q) ; y=(q \cdot P) /(k \cdot P)$, where $k, k^{\prime}$ and $P$ are the four-momenta of initial and final scattered electrons and incoming proton, respectively. These variables were reconstructed using the electron method (denoted by the subscript $e$ ), which requires measurements of the energy and angle of the scattered electron.

A three-level trigger system [12] was used to select events online. At the third level, electrons with energy greater than $4 \mathrm{GeV}$ and position outside a rectangle defined by $|\mathrm{X}|<12 \mathrm{~cm},|\mathrm{Y}|<6 \mathrm{~cm}$ on the face of the RCAL were accepted. Data below $Q^{2} \sim$ $20 \mathrm{GeV}^{2}$ were prescaled to reduce the trigger rate.

The offline selection of DIS events was based on the following requirements:

- $\left|\mathrm{Z}_{\mathrm{vtx}}\right|<50 \mathrm{~cm}$, where $\mathrm{Z}_{\mathrm{vtx}}$ is the $\mathrm{Z}$ component of the primary-vertex position determined from the tracks. This cut reduces the background from non-ep interactions;

- an identified scattered electron in the CAL with energy $E_{e} \geq 8.5 \mathrm{GeV}$;

- $2 \leq Q_{e}^{2} \leq 15000 \mathrm{GeV}^{2}$;

- $35<\delta<60 \mathrm{GeV}$, where $\delta=\sum E_{i}\left(1-\cos \theta_{i}\right), E_{i}$ is the energy of the $i^{\text {th }}$ calorimeter cell and $\theta_{i}$ is its polar angle as viewed from the primary vertex. The sum runs over all CAL cells. This cut reduces the background from photoproduction and events with large initial-state radiation;

- $y_{e} \leq 0.95$, to remove events with misidentified scattered electrons;

- $y_{\mathrm{JB}} \geq 0.04$, to remove events with low hadronic activity, where $y_{\mathrm{JB}}$ is the value of $y$ reconstructed using the Jacquet-Blondel method [21].

Good quality tracks measured in the CTD with high acceptance and resolution were selected using the following requirements: transverse momentum $p_{T}>0.15 \mathrm{GeV}$ and pseudorapidity $|\eta|<1.75$. In addition, the tracks were required to pass through more than three CTD superlayers. 
After the above cuts, the data sample contained 25 million events with at least two good tracks and the average $Q^{2}$ of the sample was $\left\langle Q^{2}\right\rangle=35 \mathrm{GeV}^{2}$.

\subsection{Selection of charged kaons}

Charged kaons were selected using the energy-loss measurement, $d E / d x$. The analysis used all tracks fitted to the primary vertex with the exception of the scattered-electron track. Tracks were selected as described above. The $d E / d x$ as a function of momentum for positively charged tracks is shown in Fig. 1a. The curves indicate the region used for identification of positively charged kaons. Kaons were selected by requiring $f<d E / d x<$ $F$, where $f$ and $F$ are functions of the track momentum, $p$, motivated by the Bethe-Bloch equation. For positive kaons $f=0.08 / p^{2}+1.0, F=0.17 / p^{2}+1.03$ mips and for negative kaons $f=0.08 / p^{2}+1.0$ and $F=0.18 / p^{2}+1.03$ mips (with $p$ in $\mathrm{GeV}$ ). The slight difference arises from the different response of the CTD to positive and negative tracks. The kaons were identified for $p<0.9 \mathrm{GeV}$ and $d E / d x>1.25$ mips.

The kaon identification efficiency for $p_{t}>0.15 \mathrm{GeV},|\eta|<1.5$ and $p<0.9 \mathrm{GeV}$ was $61 \%$, with a purity of $90 \%$. The resulting data sample contained $55522 K^{+} K^{+}$or $K^{-} K^{-}$pairs.

\subsection{Neutral kaon selection}

The $K_{S}^{0}$ mesons were identified using the charged-decay channel $K_{S}^{0} \rightarrow \pi^{+} \pi^{-}$with a similar selection as in a previous publication [22]. The pion tracks were required to originate from secondary vertices. Assigning the pion mass to both tracks, the invariant mass $M\left(\pi^{+} \pi^{-}\right)$was calculated and the candidate was accepted if the mass was within $\pm 20 \mathrm{MeV}$ of the nominal PDG [23] $K_{S}^{0}$ mass. To eliminate tracks from photon conversions and $\Lambda / \bar{\Lambda}$ contamination, the electron, pion and proton masses were assigned to tracks and the following cuts were used: $M\left(e^{+} e^{-}\right)>80 \mathrm{MeV}$ and $M(\pi p)>1121 \mathrm{MeV}$.

The following additional requirements were applied to the selected $K_{S}^{0}$ candidates:

- $2<L_{d}<30 \mathrm{~cm}$, where $L_{d}$ is the decay length of the $K_{S}^{0}$ candidate;

- $\Delta \mathrm{Z}<0.8 \mathrm{~cm}$, where $\Delta \mathrm{Z}$ is the projection on the $\mathrm{Z}$ axis of the vector defined by the primary interaction point and the point of closest approach of the $K_{S}^{0}$ candidate;

- $\alpha_{\mathrm{XY}}<8^{\circ}$, where $\alpha_{\mathrm{XY}}$ is the (collinearity) angle between the candidate $K_{S}^{0}$ momentum vector and the vector defined by the interaction point and the $K_{S}^{0}$ decay vertex in the XY plane;

- $p_{t}^{\mathrm{PA}}>0.11 \mathrm{GeV}$, where the Podolanski-Armenteros variable $p_{t}^{\mathrm{PA}}$ is the projection of the candidate pion momentum onto a plane perpendicular to the $K_{S}^{0}$ momentum direction [24]. 
The total number of $K_{S}^{0}$ candidates was 725505. After all cuts, the selected data sample contained $19494 K_{S}^{0} K_{S}^{0}$ pairs and 400 triples. Each combination of two particles was included in the analysis. Figure $1 b$ shows the $\pi^{+} \pi^{-}$invariant mass distribution after the $K_{S}^{0}$-pair selection and a fit to the signal plus linear background, which resulted in an estimated background of $1.4 \%$.

\section{Monte Carlo simulation}

Inclusive DIS events with $Q^{2}>2 \mathrm{GeV}^{2}$ were generated without BEC using the ARIADNE 4.10 Monte Carlo (MC) model [25] interfaced with HerACles 4.6.1 [26] via the DJANGOH 1.1 program $[27,28]$ in order to incorporate first-order electroweak corrections. The Lund string model [29] was used for the description of hadronisation, as implemented in the JETSET 7.4 [30] program.

The generated events were passed through a full simulation of the detector using the GEANT 3.13 program [31] and reconstructed and analyzed in the same way as the data. The ARIADnE MC sample corresponds to a similar integrated luminosity as that of the data.

\section{$5 \quad$ Extraction of BEC parameters}

The main difficulty in measuring BEC is in the construction of a reference sample which should be as close as possible to the analyzed data in all aspects but free from the BoseEinstein effect. The obvious reference sample provided by unlike-sign charged kaon pairs cannot be used due to the strong signal of the $\phi^{0}(1020) \rightarrow K^{+} K^{-}$decay at low values of $Q_{12}$.

A reference sample can be derived from a Monte Carlo simulation without BEC. In this so-called single-ratio method, the correlation function $R\left(Q_{12}\right)$ is defined as: $R^{S}\left(Q_{12}\right)=$ $P\left(Q_{12}\right)^{\text {data }} / P\left(Q_{12}\right)^{\mathrm{MC}, \text { noBEC }}$, where $P\left(Q_{12}\right)^{\text {data }}$ is the normalized two-particle density distribution for the data and $P\left(Q_{12}\right)^{\mathrm{MC}, \text { noBEC }}$ is the corresponding distribution obtained for $\mathrm{MC}$ without BEC. However, this approach requires a correct simulation of the physics processes in the absence of BEC, as well as a good description of the detector effects.

In another approach, a reference sample can be obtained using an event-mixing procedure where two kaons from different events are combined. This method, which is used in this analysis, is less sensitive to imperfections in the MC simulation. To correct for other correlations lost in the event-mixing procedure, the two-particle correlation function $R\left(Q_{12}\right)$ was calculated using the double-ratio method: 


$$
R\left(Q_{12}\right)=\frac{P\left(Q_{12}\right)^{\mathrm{data}}}{P_{\mathrm{mix}}\left(Q_{12}\right)^{\mathrm{data}}} / \frac{P\left(Q_{12}\right)^{\mathrm{MC}, \mathrm{noBEC}}}{P_{\mathrm{mix}}\left(Q_{12}\right)^{\mathrm{MC}, \mathrm{noBEC}}}
$$

where $P_{\text {mix }}\left(Q_{12}\right)^{\text {data }}$ is the two-particle density constructed from pairs of kaons coming from different events and $P_{\text {mix }}\left(Q_{12}\right)^{\mathrm{MC}, \text { noBEC }}$ is obtained in a similar way for MC events. The double-ratio method was used for the main analysis and the single-ratio method only to estimate systematic uncertainties.

To fit the correlation function $R\left(Q_{12}\right)$ defined by Eq. (4), the modified Goldhaber parametrisation (Eq. (3)) multiplied by an empirical term $1+\beta Q_{12}$, which accounts for the presence of possible long-range two-particle correlations for high $Q_{12}$, is often used:

$$
R\left(Q_{12}\right)=\alpha\left(1+\lambda e^{-Q_{12}^{2} r^{2}}\right)\left(1+\beta Q_{12}\right)
$$

Such correlations are imposed for example by energy and charge conservation, phase-space constraints or strangeness compensation. In this analysis, the $\beta$ parameter was found to be zero within errors and its possible deviation from zero was included in the systematic uncertainties.

\section{$6 \quad$ Systematic uncertainties}

Systematic uncertainties on the fitted $\lambda$ and $r$ parameters arise from event and track selection, the modeling of $d E / d x$, the fitting procedure and the construction of the reference sample. They were calculated from the deviation of the fit parameters from their nominal values after changing the analysis cuts or procedures. For charged kaons, a bias originating from the contamination of the experimental kaon sample by pion, proton and antiproton is expected. For neutral kaons, a bias can be introduced by the contamination of the sample by $K_{S}^{0} K_{S}^{0}$ pairs from the decay of $f_{0}(980)$ resonance. These effects are discussed in the next sections.

The following systematic studies were carried out for the charged kaon sample. The resulting changes for $\lambda$ and $r$ are given in parentheses as $[\Delta \lambda, \Delta r]$ :

- the correlation function was calculated using the single-ratio method $[-0.01,-0.03]$;

- the fit was repeated for different lower and upper limits of $Q_{12}$. In addition the binning in $Q_{12}$ was modified $\left[{ }_{-0.04}^{+0.04},{ }_{-0.04}^{+0.09}\right]$;

- the momentum range was reduced to $p<0.7 \mathrm{GeV}$ for both the double $[+0.06,+0.06]$ and single $[+0.01,-0.01]$ ratio methods; 
- the definition of the kaon band was varied for the double-ratio method $[-0.02,+0.05]$ and the single-ratio method $[-0.04,-0.02]$;

- the track quality cuts were changed within their resolutions $[-0.02,+0.04]$;

- the DIS selection cuts on $E_{e}, y_{e}, y_{\mathrm{JB}}, \delta$ were varied within the resolutions $\left[{ }_{-0.02}^{+0.02}{ }_{-0.02}^{+0.02}\right]$;

- the influence of pion, proton and antiproton contamination was checked by tightening and relaxing the kaon selection criteria. The purity was raised to $99 \%[+0.05,+0.07]$ and was brought down to $80 \%[-0.04,-0.05]$.

The following systematic uncertainties for neutral kaons were considered:

- the correlation function was calculated using the single-ratio method $[+0.10,+0.02]$;

- the fit was repeated for different lower and upper limits of $Q_{12}$. In addition, the binning in $Q_{12}$ was modified $\left[{ }_{-0.04}^{+0.04},{ }_{-0.04}^{+0.09}\right]$;

- the cuts on the $K_{S}^{0}$ momentum, $\alpha_{\mathrm{XY}}, \Delta \mathrm{Z}, M\left(\pi^{+} \pi^{-}\right)$were varied $[-0.08,-0.08]$;

- the cut on $p_{t}^{\mathrm{PA}}$ was changed to $0.12 \mathrm{GeV}[+0.04,+0.02]$;

- the full Eq. (5) was used to check the sensitivity of the fit to possible long-range correlations $[+0.11,+0.03]$;

- the track quality cuts were changed within the resolution $[+0.15,+0.02]$;

- the cut to remove the $e^{+} e^{-}$background was changed to $M\left(e^{+} e^{-}\right)>50 \mathrm{MeV}[+0.08,+0.03]$;

- the DIS selection cuts on $E_{e}, y_{e}, y_{\mathrm{JB}}, \delta$ were varied within resolutions $\left[{ }_{-0.02}^{+0.02}{ }_{-0.02}^{+0.02}\right]$.

The contributions from the different groups of systematic uncertainties in the parameters $\lambda$ and $r$ were added in quadrature separately for positive and negative variations. The overall systematic uncertainties in $\lambda$ and $r$ for $K^{ \pm} K^{ \pm}$and $K_{S}^{0} K_{S}^{0}$ are presented in Table 1.

\section{$7 \quad$ Results}

\subsection{Correlations in $K^{ \pm} K^{ \pm}$pairs}

Figure 2 shows the measured two-particle correlation function for $K^{ \pm} K^{ \pm}$pairs. The results obtained by the fit function given by Eq. (5) with $\beta=0$ are $r=0.57 \pm 0.09$ $\mathrm{fm}$ and $\lambda=0.31 \pm 0.06$. The $90 \%$ purity of the kaon selection introduces in the kaon pair sample an $18 \%$ admixture of unlike particles pairs which are not correlated. The measured $\lambda$ is therefore expected to be underestimated by $18 \%$. The systematic checks involving purity confirm this expectation. After the correction for purity, the result is $\lambda=0.37 \pm 0.07_{-0.08}^{+0.09}$. The value of the radius is not affected by this correction and 
the result is $r=0.57 \pm 0.09_{-0.08}^{+0.15} \mathrm{fm}$. The corrected parameters of BEC correlations for $K^{ \pm} K^{ \pm}$pairs are presented in Table 1 . The radius value is consistent with that for charged particles: $r=0.54 \pm 0.03_{-0.02}^{+0.03}$ fm for $\mathrm{H} 1$ [3] and $r=0.666 \pm 0.009_{-0.036}^{+0.022} \mathrm{fm}$ for ZEUS [4]. The obtained value of $\lambda$ for kaons is in agreement with the H1 result for charged particles $\lambda=0.32 \pm 0.02 \pm 0.06$, although somewhat smaller than the ZEUS result $\lambda=0.475 \pm 0.007_{-0.003}^{+0.011}$.

\subsection{Correlations in $K_{S}^{0} K_{S}^{0}$ pairs}

The $K_{S}^{0} K_{S}^{0}$ pairs may originate not only from $K^{0} K^{0}, \bar{K}^{0} \bar{K}^{0}$ (strangeness $S= \pm 2$ ) states but also from the $K^{0} \bar{K}^{0}$ (strangeness $S=0$ ) system, which may come from the decay of resonances. It has been shown $[32,33]$ that a Bose-Einstein-like enhancement is nevertheless expected in the $Q_{12}$ distribution of the $K_{S}^{0} K_{S}^{0}$ pairs, even when their origin is from the $K^{0} \bar{K}^{0}$ system. According to the MC simulation of DIS events, about $75 \%$ of low- $Q_{12} K_{S}^{0} K_{S}^{0}$ pairs come from $K^{0} \bar{K}^{0}$. Similar to the case of charged kaons, Eq. (5) with $\beta=0$ was used to fit the correlation function calculated for $K_{S}^{0} K_{S}^{0}$ pairs. The so-called raw results are $\lambda=1.16 \pm 0.29_{-0.08}^{+0.28}$ and $r=0.61 \pm 0.08_{-0.08}^{+0.07} \mathrm{fm}$. The results are presented in Table 1 and Fig. 3. The measured radii for $K^{ \pm} K^{ \pm}$and $K_{S}^{0} K_{S}^{0}$ are close to each other.

For $K_{S}^{0} K_{S}^{0}$ the fit does not take into account a possible contamination from the scalar $f_{0}(980)$ resonance decaying below the $K \bar{K}$ threshold. The decay channel $f_{0}(980) \rightarrow K^{0} \bar{K}^{0}$ is not included in the standard MC, therefore the $f_{0}(980)$ contribution may distort the strength of the Bose-Einstein effect. In order to investivate this, a MC sample with BEC at maximum strength was generated. A comparison of this $\mathrm{MC}$ with the data showed an excess for $Q_{12}<0.6 \mathrm{GeV}$, which may indicate a possible $f_{0}$ contribution. The same excess is also visible in the $M_{K K}$ distribution, as illustrated in Fig. 4a.

The expected contribution from $f_{0}$ can be approximated by a modified Breit-Wigner function as proposed by Flatté [34-36]:

$$
\frac{d \sigma}{d M_{K K}}=N_{F} \cdot \frac{m_{0}^{2} \cdot \Gamma_{K K}}{\left(m_{0}^{2}-M_{K K}^{2}\right)^{2}+\left(m_{0} \cdot\left(\Gamma_{\pi \pi}+\Gamma_{K K}\right)\right)^{2}}
$$

where $m_{0}=0.954 \mathrm{GeV}$ is the mass of $f_{0}(980)$ and the widths $\Gamma_{\pi \pi}$ and $\Gamma_{K K}$ are related to the coupling constants $g_{\pi}=0.11$ and $g_{K}=0.423$ by $\Gamma_{\pi \pi}=g_{\pi} \sqrt{M_{K K}^{2} / 4-m_{\pi}^{2}}$ and $\Gamma_{K K}=g_{K} \sqrt{M_{K K}^{2} / 4-m_{K}^{2}}$. The normalization factor, $N_{F}$, was adjusted to give the total number of $f_{0}(980) \rightarrow K_{S}^{0} K_{S}^{0}$ decays for $M_{K K}<1.8 \mathrm{GeV}$. Fig. 4a shows the distribution of $d \sigma / d M_{K K}$ according to Eq. (6). Figure $4 \mathrm{~b}$ shows the $M_{K K}$ distribution in the data after subtraction of the standard MC without BEC together with different contributions of the $f_{0}$ resonance. An $f_{0}$ contribution of about $7 \%$ is sufficient to account for the data, 
therefore, the present analysis cannot distinguish the Bose-Einstein effect from the effect of an $f_{0}$ contribution. Assuming that both BEC and $f_{0}$ are present, the amount of $f_{0}$ was estimated from the shape of the $R\left(Q_{12}\right)$ distribution (Eq. (4)). For this purpose, fits of the correlation function were carried out with different percentages, $c_{f_{0}}$, of $f_{0}$ subtracted from the data. A shallow minimum of the $\chi^{2}\left(c_{f_{0}}\right)$ value for $c_{f_{0}}=4 \%$ was found, with one-sigma limits of $c_{f_{0}}=1 \%$ and $c_{f_{0}}=7 \%$. The values of $\lambda$ and $r$ at $c_{f_{0}}=4 \%$ were taken as the most probable result and their uncertainties were calculated from the one sigma limits of $c_{f_{0}}$.

The results corrected for the $f_{0}$ contamination are included in Table 1. After adding the uncertainties coming from the $f_{0}$ subtraction to systematics, the corrected results are $\lambda=0.70 \pm 0.19_{-0.53}^{+0.47}$ and $r=0.63 \pm 0.09_{-0.08}^{+0.11} \mathrm{fm}$. The uncertainty on a possible $f_{0}$ admixture leads to a large systematic uncertainty on $\lambda$. The radius $r$ is less sensitive to the $f_{0}$ admixture. The radii for $K_{S}^{0} K_{S}^{0}$ and $K^{ \pm} K^{ \pm}$are very similar.

\subsection{Comparison with $e^{+} e^{-}$interactions}

Figure 5 shows the comparison for $r$ between DIS and $e^{+} e^{-}$annihilation results at LEP [36-39] for both charged and neutral kaons. The radius value obtained in DIS agrees with the measurements from LEP. The same figure also presents DIS results for unidentified charged particles. The kaon results agree with those for charged particles within systematic uncertainties. The $\lambda$ value for charged kaons in DIS is somewhat smaller than that in $e^{+} e^{-}$collisions, which may be related to the fact that the DIS data mostly populate the proton fragmentation region in the Breit frame.

\section{Conclusions}

Bose-Einstein correlations have been measured for pairs of charged and neutral kaons in deep inelastic scattering at HERA using the ZEUS detector. The values of the radius for charged and neutral kaons agree within systematic uncertainties. They are also consistent with the measurements for charged particles in DIS and kaons in $e^{+} e^{-}$collisions at LEP. The $f_{0}(980) \rightarrow K_{S}^{0} K_{S}^{0}$ decay can significantly affect the $\lambda$ parameter for $K_{S}^{0} K_{S}^{0}$ correlations.

\section{Acknowledgments}

We wish to thank the DESY Directorate for their strong support and encouragement. The remarkable achievements of the HERA machine group were essential for the successful 
completion of this work and are greatly appreciated. We are grateful for the support of the DESY computing and network services. The design, construction and installation of the ZEUS detector have been made possible owing to the ingenuity and effort of many people from DESY and home institutes who are not listed as authors. 


\section{References}

[1] G. Goldhaber et al., Phys. Rev. Lett. 3, 181 (1959).

[2] G. Goldhaber et al., Phys. Rev. 120, 300 (1960).

[3] H1 Coll., C. Adloff et al., Z. Phys. C. 75, 437 (1997).

[4] ZEUS Coll., S. Chekanov et al., Phys. Lett. B 583, 231 (2004).

[5] G. Alexander, Rep. Prog. Phys. 66, 481 (2003).

[6] T. Csörgo, Acta Phys. Hung. A 15, 1 (2002).

[7] T. Csörgo. J. Phys. Conf. Ser. 50:259-270 (2006), Preprint nucl-th/0505019.

[8] R. M. Weiner, Introduction to Bose-Einstein Correlations and Subatomic Interferometry. John Wiley \& Sons, 2000.

[9] W. Kittel and E. A. De Wolf, Soft multihadron dynamics. World Scientific Singapore, 2005.

[10] ZEUS Coll., J. Breitweg et al., Eur. Phys. J. C 11, 251 (1999).

[11] R. J. Glauber, Phys. Rev. Lett. 10, 84 (1963).

[12] ZEUS Coll., U. Holm (ed.), The ZEUS Detector. Status Report (unpublished), DESY (1993), available on http://www-zeus.desy.de/bluebook/bluebook.html.

[13] N. Harnew et al., Nucl. Inst. Meth. A 279, 290 (1989);

B. Foster et al., Nucl. Phys. Proc. Suppl. B 32, 181 (1993);

B. Foster et al., Nucl. Inst. Meth. A 338, 254 (1994).

[14] ZEUS Coll., J. Breitweg et al., Phys. Lett. B 481, 213 (2000).

[15] ZEUS Coll., J. Breitweg et al., Eur. Phys. J. C 18, 625 (2001).

[16] D. Bartsch. PhD thesis (unpublished), Bonn University, 2007.

[17] M. Derrick et al., Nucl. Inst. Meth. A 309, 77 (1991);

A. Andresen et al., Nucl. Inst. Meth. A 309, 101 (1991);

A. Caldwell et al., Nucl. Inst. Meth. A 321, 356 (1992);

A. Bernstein et al., Nucl. Inst. Meth. A 336, 23 (1993).

[18] ZEUS Coll., S. Chekanov et al., Eur. Phys. J. C 21, 443 (2001).

[19] A. Bamberger et al., Nucl. Instrum. Methods A 401, 63 (1997).

[20] A. Bamberger et al., Nucl. Inst. Meth. A 382, 419 (1996).

[21] F. Jacquet and A. Blondel, U. Amaldi (ed.) DESY, Germany, p. 391 (1979).

[22] ZEUS Coll., S. Chekanov et al., Phys. Lett. B 591, 7 (2004). 
[23] Particle Data Group, W-M Yao et al., J. Phys. G 33, 1 (2006).

[24] J. Podolanski and R. Armenteros, Philos. Mag. 45, 13 (1954).

[25] L. Lönnblad, Comp. Phys. Comm. 71, 15 (1992).

[26] A. Kwiatkowski, H. Spiesberger and H.-J. Möhring, Comp. Phys. Comm. 69, 155 (1992).

[27] K. Charchula, G.A. Schuler and H. Spiesberger, Comp. Phys. Comm. 81, 381 (1994).

[28] H. Spiesberger, DJANGOH and HERACLES Monte Carlo programs, available on http://www.desy.de/ hspiesb/mcp.html.

[29] B. Andersson et al., Phys. Rep. 97, 31 (1983).

[30] T. Sjöstrand, Comp. Phys. Comm. 82, 74 (1994).

[31] R. Brun et al., CERN-DD/EE/ 84-1 (1987).

[32] H. J. Lipkin, Phys. Lett. B 219, 474 (1989).

[33] H. J. Lipkin, Phys. Rev. Lett. 69, 3700 (1989).

[34] S. M. Flatté, Phys. Lett. B 63, 224 (1976).

[35] S. M. Flatté, Phys. Lett. B 63, 228 (1976).

[36] ALEPH Coll., S. Schael et al., Phys. Lett. B 611, 66 (2005).

[37] DELPHI Coll., S. Abreu et al., Phys. Lett. B 379, 330 (1996).

[38] OPAL Coll., G. Abbiendi et al., Eur. Phys. J. C 21, 23 (2001).

[39] OPAL Coll., R. Akers et al., Z. Phys. C. 67, 389 (1995). 


\begin{tabular}{|c|c|c|}
\hline & $\lambda$ & $r[\mathrm{fm}]$ \\
\hline$K^{ \pm} K^{ \pm}$(corrected) & $0.37 \pm 0.07_{-0.08}^{+0.09}$ & $0.57 \pm 0.09_{-0.08}^{+0.15}$ \\
$K_{S}^{0} K_{S}^{0}$ (raw) & $1.16 \pm 0.29_{-0.08}^{+0.28}$ & $0.61 \pm 0.08_{-0.08}^{+0.07}$ \\
$K_{S}^{0} K_{S}^{0}$ (corrected) & $0.70 \pm 0.19_{-0.08-0.52}^{+0.28+0.38}$ & $0.63 \pm 0.09_{-0.08-0.02}^{+0.07+0.09}$ \\
\hline
\end{tabular}

Table 1: The radius $r$ and the correlation strength $\lambda$ for charged and neutral kaons extracted from fitting the Goldhaber parametrization (Eq. (5) with $\beta=0)$ to the Bose-Einstein correlation function. The first uncertainties are statistical and the second systematic. The raw results for $K_{S}^{0} K_{S}^{0}$ pairs correspond to those shown in Fig. 3; the corrected results were obtained after subtraction of the $f_{0}$ contribution from the data. For the corrected values the third uncertanty comes from the $f_{0}$ subtraction. 
ZEUS
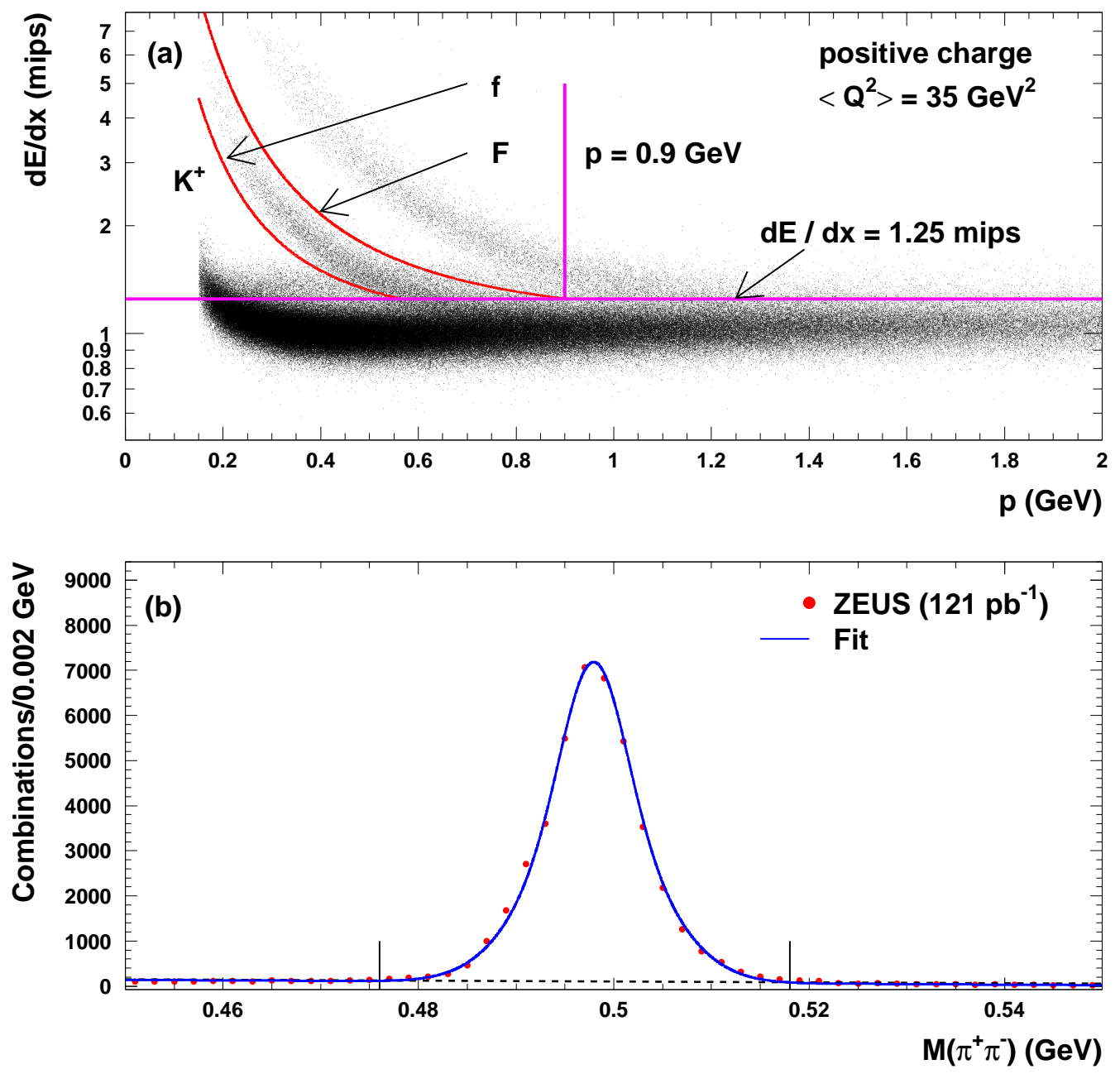

Figure 1: (a) The energy-loss $d E / d x$ as a function of the momentum $p$ for tracks with positive charge. The tracks with $f<d E / d x<F, d E / d x>1.25$ mips and $p<0.9 \mathrm{GeV}$ were taken as $K^{+}$. (b) The $\pi^{+} \pi^{-}$invariant-mass distribution of the $K_{S}^{0}$ candidates. The solid line shows the result of a fit with the sum of two Gaussian functions and linear background. The two Gaussians were fixed to the same mean value. A mass in agreement with the Particle Data Group value [23] and a width of $3.2 \mathrm{MeV}$ (6.3 MeV) were obtained from the fit for the central (second) Gaussian. The dashed line shows the linear background. The short vertical lines show the mass window used to define the signal. 


\section{ZEUS}

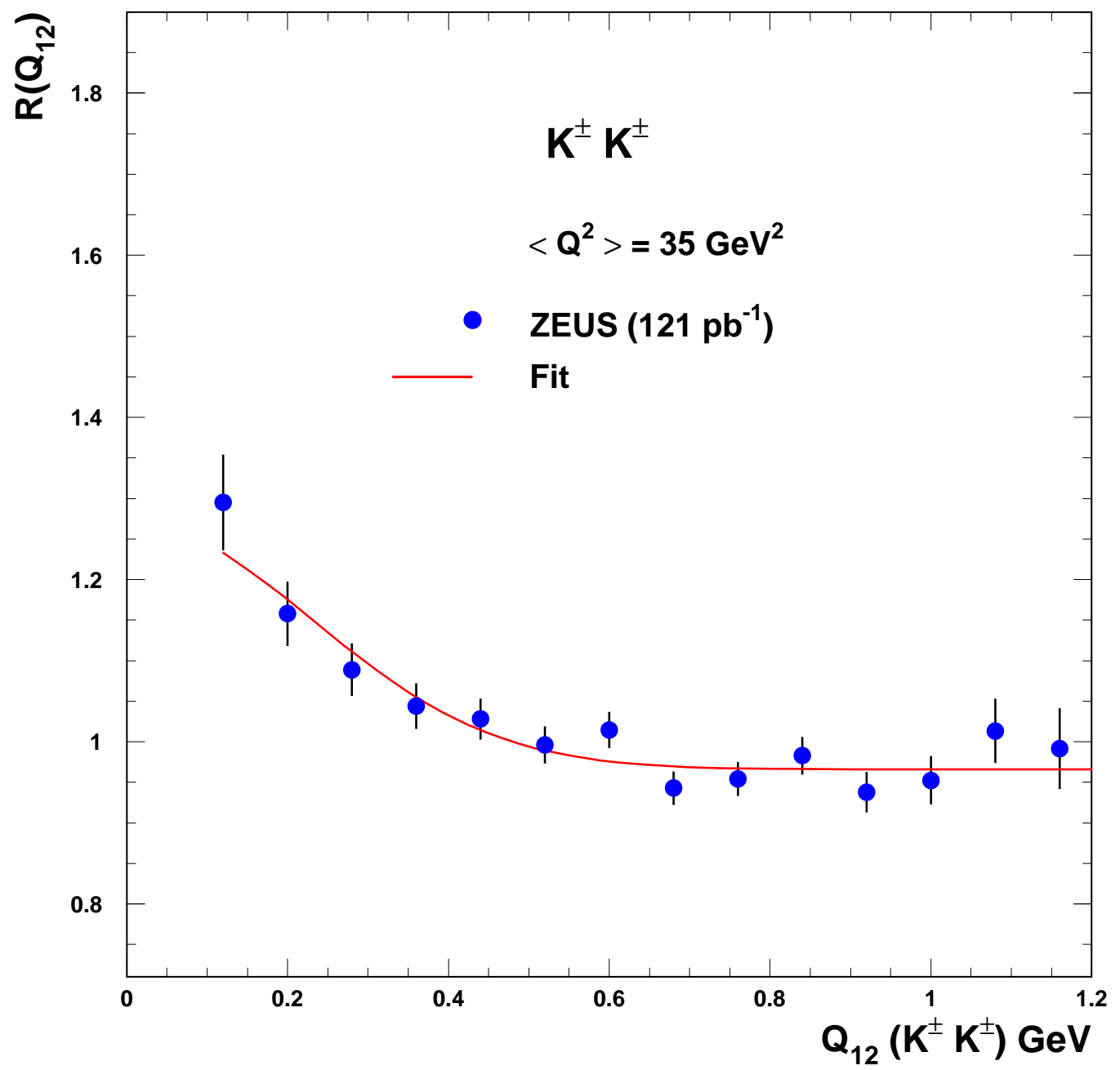

Figure 2: The two-particle correlation function for charged kaons with a fit to Eq. (5), with $\beta=0$. The error bars represent the statistical uncertainties. 


\section{ZEUS}

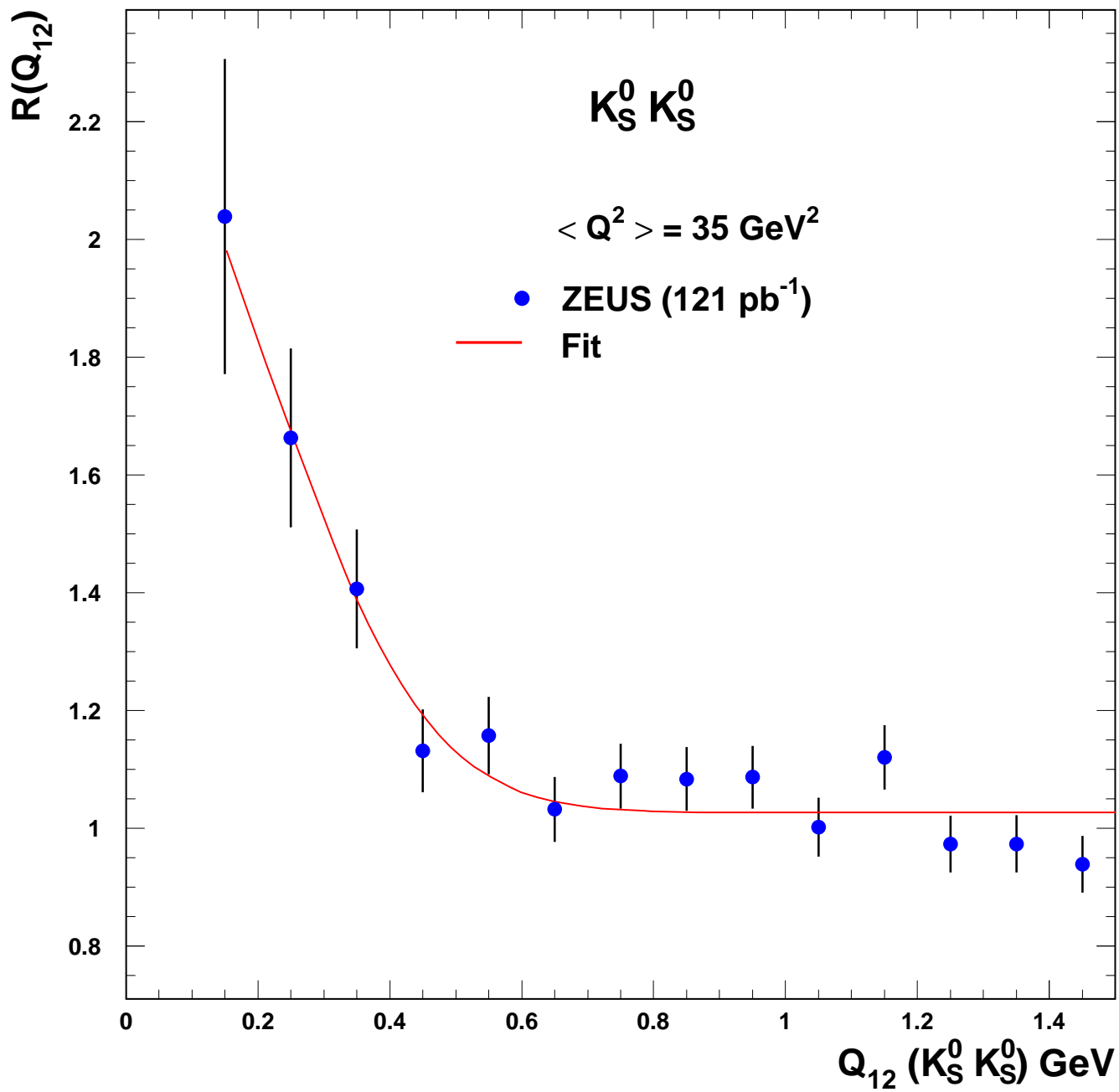

Figure 3: The two-particle correlation function for neutral kaons with a fit to Eq. (5), with $\beta=0$. The error bars represent the statistical uncertainties. 


\section{ZEUS}

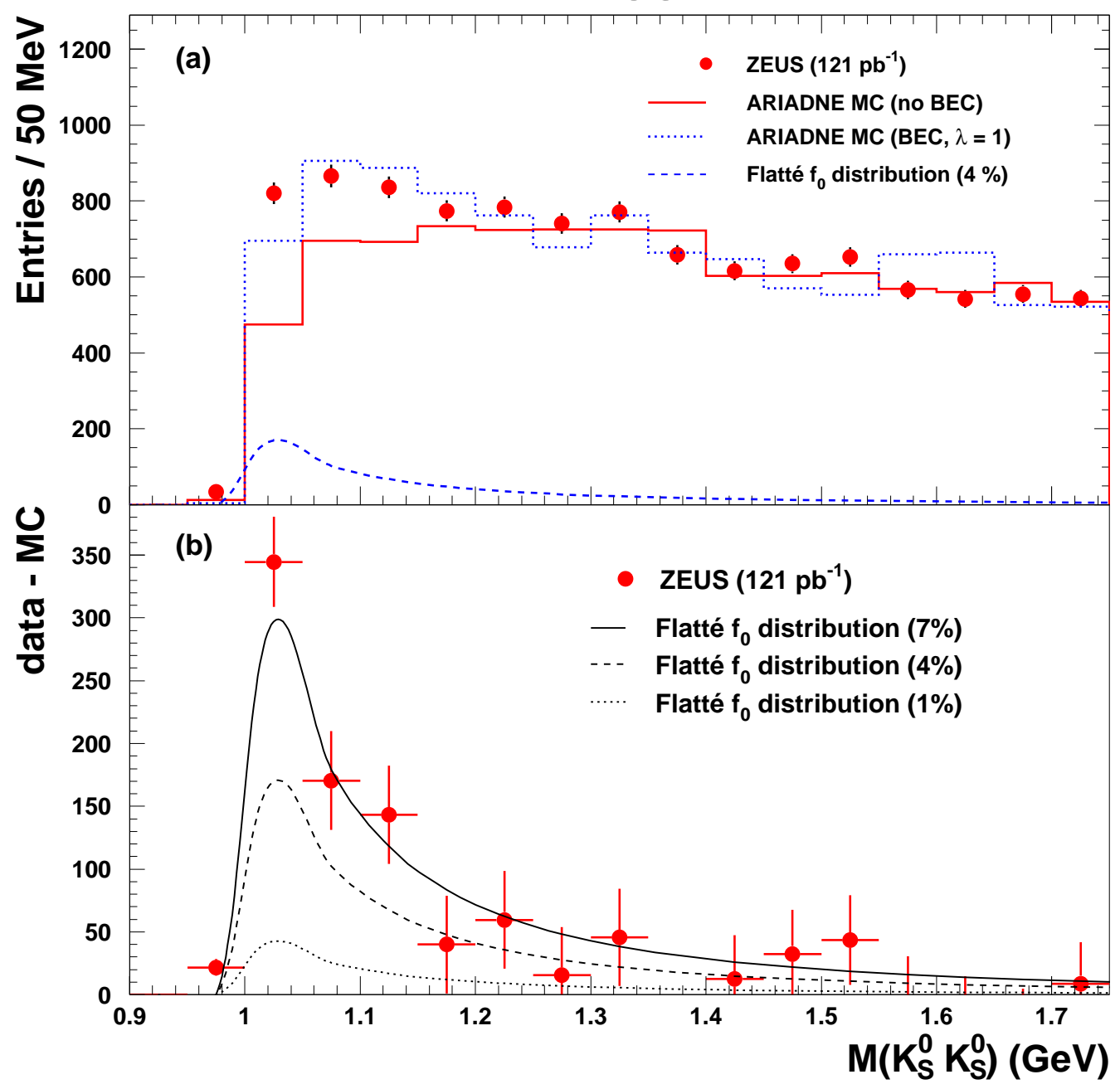

Figure 4: (a) $K_{S}^{0} K_{S}^{0}$ invariant mass for data (points) and Monte Carlo with BEC (dotted histogram) and without BEC (full-line histogram). The dashed line shows the Flatté function used to describe the $f_{0}$ resonance. (b) The difference between data and $M C$ without BEC compared to different $f_{0}$ contributions. 


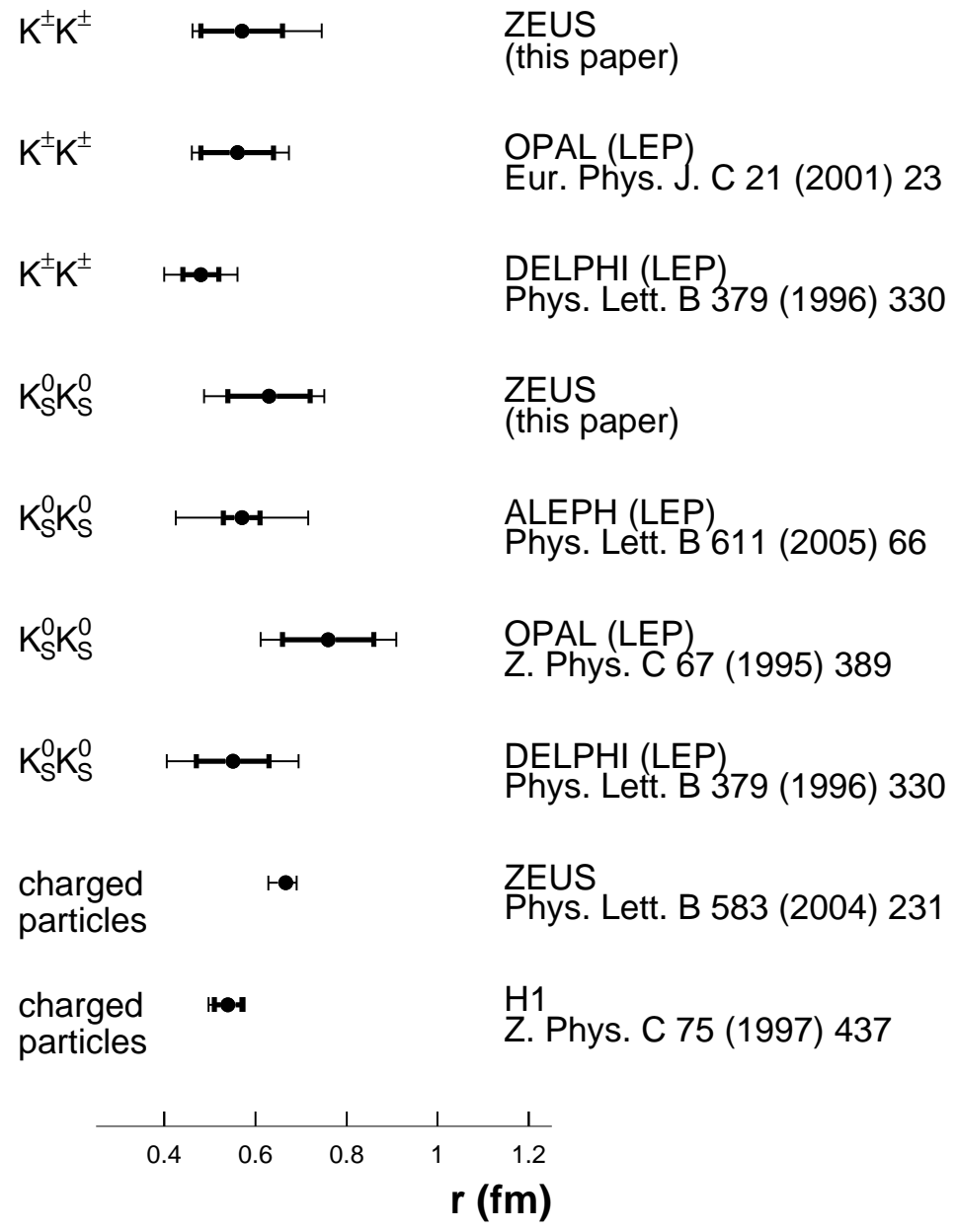

Figure 5: Comparison of DIS and LEP results for $r$ obtained from Bose-Einstein correlation studies of charged and neutral kaons and unidentified charged particles. The DIS result for neutral kaons is corrected for the $f_{0}$ contribution. 